\title{
Enhancing the prediction accuracy of bovine lameness models through transformations of limb movement variables
}

\author{
J. Liu, ${ }^{*}$ N. K. Neerchal, † U. Tasch,,${ }^{\star 1}$ R. M. Dyer, $\ddagger$ and P. G. Rajkondawar§ \\ *Department of Mechanical Engineering, and \\ †Department of Mathematics and Statistics, University of Maryland, Baltimore County, Baltimore 21250 \\ łDepartment of Animal and Food Sciences, University of Delaware, Newark 19717 \\ §Bou-Matic LLC, Madison, WI 53708
}

\section{ABSTRACT}

The issue of modeling bovine lameness was explored by testing the hypothesis that B-spline transformation of limb movement variables (LMV) employed in predictive models improved model accuracy. The objectives were to determine the effect of number of B-spline knots and the degree of the underlying polynomial approximation (degree of freedom) on model accuracy. Knot number used in B-spline transformation improved model accuracy by improving model specificity and to a lesser extent model sensitivity. Degree of polynomial approximation had no effect on model predictive accuracy from the data set of 261 cows. Model stability, defined as changes in predictive accuracy associated with the superimposition of perturbations (0.5 and $1.0 \%)$ in LMV on the measured data, was explored. Model specificity and to a lesser degree, sensitivity, increased with increased knot number across data set perturbations. Specificity and sensitivity increased by 43 and $11 \%$, respectively, when knot number increased from 0 to 7 for a perturbation level of $0.5 \%$. When the perturbation level was $1 \%$, the corresponding increases in specificity and sensitivity were 32 and $4 \%$, respectively. Nevertheless, different levels of LMV perturbation varied the optimal knot number associated with highest model accuracy. The optimal knot number for $0.5 \%$ perturbation was 8 , whereas for $1 \%$ perturbation the optimal knot number was 7 . The B-spline transformation improved specificity and sensitivity of predictive models for lameness, provided the appropriate number of knots was selected.

Key words: dairy cattle, lameness, limb movement variable, spline transformation

Received April 25, 2008

Accepted January 19, 2009.

${ }^{1}$ Corresponding author: tasch@umbc.edu

\section{INTRODUCTION}

Lameness is an important welfare issue and a costly problem to the dairy industry (Animal Welfare Foundation, 1993-1994). Early detection of bovine lameness enables the farmer to provide effective treatment that could improve the effectiveness of managing hoof and leg ailments (Green et al., 2002). This would therefore benefit both the welfare of dairy cows and the profitability of the dairy industry by reducing lameness-associated losses in milk yields (Amory et al., 2008; Bicalho et al., 2008) and involuntary culling (Booth et al., 2004; Espejo et al., 2006), and by enhancing reproductive efficiency (Garbarino et al., 2004; Bicalho et al., 2007b). In spite of efforts to control lameness, recent prevalence estimates from the National Animal Health Monitoring System showed that the prevalence increased from $10.5 \%$ in 1996 to $14.0 \%$ in 2007 and was currently second only to mastitis in disease problems on modern dairy farms (USDA, 2008). Reasons for this undesirable trend remained unclear, but may be related, in part, to producer insensitivity toward lameness, detection techniques with insensitive diagnostic abilities, or increased labor problems resulting from expanding herd size in light of diminishing labor supply (Whay et al., 2003). Cost-effective management of bovine lameness should benefit from early, accurate diagnostic techniques. Development of objective, automatic lameness scoring systems that provide continuous monitoring would be of great practical and economic value to the industry.

Minimum observer qualifications for early lameness detection include individuals with accurate diagnostic abilities and the time to invest in individual gait assessment. Many reports showed visual gait analysis suffers from a lack of sensitivity, specificity, and reproducibility, making visual observation an unreliable method of detection (Espejo et al., 2006; Flower and Weary, 2006; Bicalho et al., 2007a). Much of the problem likely stems from the subjective nature of gait evaluation, which is particularly problematic at mild to moderate levels of lameness. Because visual examination requires substan- 
tial time investment in light of shrinking labor pools, early detection suffers from delayed diagnosis.

Automated methods of detection based on kinetic or kinematic data could provide objective evaluations of gait on a continuous daily basis, overcoming many problems associated with visual inspection. To be useful, automated detection must provide diagnostic information with high sensitivity, specificity, and reproducibility. An automatic lameness scoring system recently introduced to the industry (StepMetrix, BouMatic, Madison WI) was based upon detection of kinetic measures of a clinical evaluation (Rajkondawar et al., 2001). StepMetrix, in its current version, predicted lameness probabilities of the hind limbs through an algorithm that equated the dependent explanatory variable, clinical lameness diagnosis, to a series of independent explanatory limb movement variables (LMV) generated from kinetic outputs. This model calculated the probability that one of the hind limbs was lame. An early field trial (Bicalho et al., 2007a) indicated that the model misclassified lame animals, while correctly classifying sound animals. In an effort to improve the model performance, the impact of LMV transformation on model predictions was explored. There are many approaches available to handle nonlinear transformations of predictor variables. There is literature on such methods in the statistical research area, known as nonparametric regression (Eubank, 1999). B-spline transformations (Schumaker, 2007) were considered. The transformed LMV were determined by a family of B-spline transformations. Spline transformations have 2 main parameters that must be determined to improve model accuracy: the number of knots employed in the transformation, and the degree of freedom employed in the underlying polynomial approximation.

The objectives of this study were 1) to evaluate the effect of B-spline LMV transformation on the prediction accuracy of a logistic regression model, where LMV and the clinical lameness index are independent and dependent variables, respectively; 2) to characterize the effect of LMV variability on model predictions; and 3) to determine the effects of number of knots and degrees of freedom on accuracy of model predictions. We hypothesized that B-spline transformation of the LMV would improve lameness model performance as determined by model sensitivity and specificity.

\section{MATERIALS AND METHODS}

\section{Animals Used to Generate the Data Set}

The LMV data used to generate the models were derived from 261 cows residing in 2 commercial dairy herds that consist of 550 and 1,450 lactating dairy cows, respectively. Out of the 261 cows, 63 were sound and 198 were mildly lame or lame. The LMV data of 145 cows from the former herd and 116 from the latter herd were collected. Housing on each farm consisted of curtained free-stall barns with concrete alleys and stalls with rubber mattresses. Cattle were milked 3 times daily in a parlor, thereby enabling data collection 3 times daily because StepMetrix was located in the parlor exit alley. Cows in both herds were fed a TMR 3 times daily consisting of corn silage, alfalfa or rye haylage, high-moisture corn, soybean meal or roasted soybeans, cottonseed, and fat and mineral supplements. Rations were formulated to meet NRC requirements of a $665-\mathrm{kg}$ cow milking $38 \mathrm{~kg}, 3.5 \% \mathrm{FCM}$ per day (NRC, 2001).

\section{Veterinary Examination}

Cows were examined by a veterinarian $1 \mathrm{~d}$ after collecting the LMV data. Locomotion scores were established for each cow by a single veterinarian as described by Dyer et al. (2007). Every week, 12 to 18 randomly selected cows were observed at a stance, while walking in a straight line, and while circled to the right and to the left. All evaluations were performed in an alleyway of solid, concrete flooring free of slurry and lightly covered with wood shavings. Locomotion observations were performed perpendicular, parallel, posterior, and anterior to the line of travel. Cows and limbs were observed for freedom of motion; stride length of the left and right pectoral and pelvic limbs; length of anterior and posterior swing phases; symmetry and arc of the foot flight; foot placement relative to body position and limb axis; foot rotation during weight bearing; symmetry of weight distribution at the walk and stance; and position of top line at a walk and stance. A score of 1 (sound) to 5 (severely lame) was assigned for each limb.

Cows were placed in a chute equipped with a head gate and a manually operated rope foot lift (farm 1). Pain evaluation, trimming, and lesion diagnosis followed limb restraint by manually elevating the limb with a rope fastened mid-diaphysis of the third metatarsal bone. On farm 2, cows were placed on a hydraulic table and positioned into lateral recumbency. All feet were cleaned and trimmed to remove stained, overgrown hoof horn tissue following guidelines of Raven (1985). Pain evaluations and lesion diagnoses were performed as described in Dyer et al. (2007).

Claw pain was evaluated through claw compression using a hoof tester equipped with a Dillon force gauge (Dillon model X force gauge 250, Dillon Force Measurement Products and Systems, Fairmont, MN). Cows were initially adapted to the process of hoof compres- 
Table 1. Definitions of the 4 limb movement variables (LMV)

\begin{tabular}{lll}
\hline LMV & Unit & Description \\
\hline PGRF & Nondimensional & Peak ground reaction force (Newton) normalized with respect to the BW of a cow \\
AGRF & Nondimensional & Average ground reaction force (Newton) normalized with respect to the BW of a cow \\
ST & $\mathrm{s}$ & Stance time is the time during which a limb is in contact with the floor plate \\
$\mathrm{GRF}_{\omega}$ & $1 / \mathrm{s}$ & The integral of the magnitude of the ground reaction force of an individual limb with respect to frequency $\omega$ \\
\hline
\end{tabular}

sion, and then pain determinations were performed along the axis extending between abaxial and axial walls through regions 3,4 , and 5 of the claw (Shearer et al., 2004). Pain indices were calculated as $\frac{P_{i}}{P_{\max }}$ (dimensionless), where $P_{i}$ was the pressure recorded upon limb withdrawal and $P_{\max }$ was $459.74 \mathrm{~N} / \mathrm{cm}^{2}$.

Pain associated with integument lesions was assessed by an algometer (44.48 N scale; Wagner Force Dial FDK 10, Wagner Instruments, Greenwich, CT) with a blunt probe. Integument pain indices were calculated as $\frac{P_{i}}{P_{\max }}$, where $P_{i}$ was the pressure recorded upon limb withdrawal and $P_{\max }$ was $140.54 \mathrm{~N} / \mathrm{cm}^{2}$. The final pain index for the limb was determined as the smaller $\frac{P_{i}}{P_{\max }}$ of the claws and interdigital space. Any $\frac{P_{i}}{P_{\max }}<1.0$ was considered indicative of a pain response.

Lameness was ascribed to a limb when a lesion was present, or the limb had a positive pain response upon compression of the soft tissues or claws, and the gait (cow level) was scored higher or equal to 2. Lameness was modeled at 3 levels: individual limb, combination of the 2 pelvic or the 2 pectoral limbs, and the entire cow level. In the limb level, when at least one of the forelimbs was lame, the cow was classified as a forelimb lameness, and when at least one of the hindlimbs was lame, the cow was classified as a hindlimb lameness. The cow was classified lame when at least 1 of the 4 limbs was determined lame. All procedures employed in this work were pre-approved by the Agricultural Animal Care and Use Committee in the Department of Animal and Food Sciences, College of Agriculture and Natural Resources, University of Delaware.

\section{LMV}

The signals of 8 load cells were recorded as a cow walked through the StepMetrix (Rajkondawar et al., 2001). These signals were processed and 4 LMV (peak ground reaction force, PGRF; average ground reaction force; AGRF; stance time, $\mathbf{S T}$; and ground reaction force integral $\mathbf{G R F}_{\omega}$; Table 1) were recorded for each limb. The PGRF and AGRF were normalized with respect to the BW, whereas the dimensions of ST and $\mathrm{GRF}_{\omega}$ were s and $1 / \mathrm{s}$, respectively. Hence, 16 variables characterized the gait of a single cow. Nonlinear spline transformations (Schumaker, 2007) were performed on the $16 \mathrm{LMV}$ for modeling purposes.

\section{Lameness Models: Logistic Regression}

An early lameness prediction model (Rajkondawar et al., 2002) was employed based on logistic regression (Hosmer and Lemeshaw, 2000) to predict the probability of lameness as a function of the LMV. That is,

$$
P(\text { Lameness }=1)=\frac{e^{\beta_{0}+\Sigma \beta_{i} L M V_{i}}}{1+e^{\beta_{0}+\Sigma \beta_{i} L M V_{i}}},
$$

where the $\beta$ coefficients are estimated by appropriate statistical methods and $\operatorname{LMV}_{i}(i=1, \ldots, 16)$ is the $i$ th LMV measurement. Predictions from the logistic regression models were converted into lameness scores ranging from 0 to 100 . In addition, the LMV were transformed using spline expansions (Schumaker, 2007). An implementation of these transformations was available in the statistical software (PROC TRANSREG; SAS Institute, 2004). B-spline transformation expanded a single input variable into multiple (knots + degree +1 ) outputs. The output variables constituted the spline basis that was used internally to generate a transformed value for each LMV. The transformed LMV were denoted by TLMV; thus, the new model was of the form:

$$
P(\text { Lameness }=1)=\frac{e^{\beta_{0}+\Sigma \beta_{i} T L M V_{i}}}{1+e^{\beta_{0}+\Sigma \beta_{i} T L M V_{i}}} .
$$

Note that Equation [2] can be applied to construct a model at the cow level, the individual limb level, or separately for the fore- and hindlimbs.

\section{Evaluation of Model Performance by Cross Validation}

The leave-one-out (Kohavi, 1995) method of cross validation was used to evaluate the performance of the 
lameness models. In this cross-validation method, a single observation from the original data set was used as the validation data, and the remaining observations served as the training data. This exercise was repeated until every observation in the sample was used once as validation data. Using this leave-one-out method, the model was reconstructed 261 times, where a single data input (1 cow) was omitted and the probability that the omitted cow was lame was calculated with the model constructed by the other 260 data points.

\section{Receiver Operating Characteristic Curve}

Receiver operating characteristic (ROC) curves provided a method of measuring model accuracy (Hosmer and Lemeshow, 2000). For a fixed trial threshold value of c, the sensitivity (probability of detecting the true signal) was computed as the proportion of truly lame cows for which the predicted probability of lameness exceeded c. Similarly, specificity (the probability of detecting the false signal) was computed as the truly sound cows for which the predicted probability was below $\mathrm{c}$. The ROC was obtained from plotting the sensitivity against (1- specificity), as c increased from 0 to 1 . The area under the ROC curve (AUC) was interpreted as the probability of the overall true diagnosis.

\section{Testing the Lameness Model on Simulated Data}

Experience showed the LMV data contained noise because of the inherent variability across multiple trials of evaluated gaits at a walk. A computer experiment was performed to simulate the variability in the data and evaluate the degree of deterioration in model performance as the data set was perturbed. This intuitive concept was used to determine an "optimal" number of knots.

To train the lameness model, the TLMV of the original 261 cows were used as independent explanatory data along with their clinical diagnoses, which were used as dependent variables. This resulted in a lameness model that used TLMV. The model was tested by perturbing the original untransformed LMV. For a given cow there were 16 original untransformed LMV that were perturbed with randomly generated, normally distributed perturbations. Each perturbed $\mathrm{LMV}_{\mathrm{i}}$ was a sum of 2 contributors: 1 ) the original measured $\mathrm{LMV}_{\mathrm{i}}$ that contained measurement noise, and 2) artificial perturbation $\varepsilon_{\mathrm{i}}$ that was proportional to the standard deviation of $\mathrm{LMV}_{\mathrm{i}}$. This was expressed mathematically as follows:

$$
\text { Perturbed_LMV } V_{i}=L M V_{i}+\varepsilon_{i}
$$

$$
\varepsilon_{i}=0.01 \cdot S D\left(L M V_{i}\right) \cdot \operatorname{RANNOR}(0,1)
$$

where $S D\left(L M V_{i}\right)$ is the standard deviation of the $i$ th LMV $(i=1, \ldots, 16)$ for the 261 cows, $L M V_{i}$ is the $i$ th LMV measurement for the given cow, and $R A N N O R(0$, 1 ) is a normally distributed random number with mean $=0$ and standard deviation $=1$.

This process was repeated 100 times for every cow. Thus, from the original 261 cows a database was generated that represented 26,100 cows. The nonlinear transformation was applied for the LMV of this database. The 26,100 perturbed LMV were then transformed using spline transformation, and these data along with the clinical diagnoses were used as the testing database to predict the clinical diagnoses. By comparing the predicted and actual clinical diagnoses the accuracy of the model was evaluated. By plotting the accuracy of the model predictions when the perturbed LMV were used versus the number of knots, we identified the number of knots that warranted a high level of satisfactory accuracy of the lameness prediction for the simulated cow database.

\section{RESULTS}

\section{Lameness Models at the Limb and Cow Levels}

Because lameness models were developed at the limb and cow level, the accuracy of performances (percentage correct diagnoses, sensitivity, and specificity) was determined for both the limb and cow level models. The limb lameness models were derived for the fore- and hind limbs and evaluated by the leave-one-out method (Table 2 ). Note both limb- and cow-level model performance using untransformed LMV was marked by a correct classification 71.3 and $72.8 \%$ of the time, respectively. Limb and cow models performed with a high sensitivity ( 88.6 and $89.9 \%$, respectively) but low specificity (5.8 and $19.1 \%$, respectively). Accordingly, the AUC value for the cow level model was low $(69.7 \%)$ when untransformed LMV were employed. Use of spline-transformed LMV in the hind limb model (knots 1 to 15, Table 2 ) increased the percentage of correct diagnoses from 69.0 to $98.5 \%$, increased sensitivity from 85.0 to $99.0 \%$, and increased specificity from 23.5 to $97.0 \%$. Similarly, use of spline-transformed LMV in the cow-level model increased the percentage of correct diagnoses from 73.2 to $99.6 \%$, increased sensitivity from 89.4 to $99.5 \%$, and increased specificity from 22.2 to $100.0 \%$ as the number of knots increased from 1 to 15 . Moreover, AUC values for the cow level model increased from 73.0 to $99.2 \%$ as the number of knots in the spline transformation increased from 1 to 15 . Of note, spline transforma- 
Table 2. Correct lameness diagnosis and area under the curve (AUC) for models at the limb (fore and hind) and cow levels, as a function of number of knots, when degrees of freedom $=1^{1}$

\begin{tabular}{lccc}
\hline Knots, $\mathrm{n}$ & $\begin{array}{c}\text { Correct diagnosis at the limb } \\
\text { level for the forelimbs, } \%\end{array}$ & $\begin{array}{c}\text { Correct diagnosis at the limb } \\
\text { level for the hind limbs, } \%\end{array}$ & $\begin{array}{c}\text { Correct diagnosis at } \\
\text { the cow level, } \%\end{array}$ \\
\hline 0 & $89.7(0,99.2)$ & $71.3(88.6,5.8)$ & $\begin{array}{c}\text { AUC at the } \\
\text { cow level, } \%\end{array}$ \\
1 & $89.3(16.0,97.0)$ & $69.0(85.0,23.5)$ & $72.8(89.9,19.1)$ \\
2 & $89.7(20.0,97.0)$ & $76.2(87.6,44.1)$ & $73.2(89.4,22.2)$ \\
3 & $88.5(28.0,94.9)$ & $78.2(88.6,48.5)$ & $78.9(89.9,44.4)$ \\
4 & $90.0(36.0,95.8)$ & $79.3(89.6,50.0)$ & $77.8(89.4,41.3)$ \\
5 & $91.2(44.0,96.2)$ & $80.5(88.6,57.4)$ & $78.9(89.4,44.4)$ \\
6 & $93.5(64.0,96.6)$ & $83.5(90.7,63.2)$ & $81.6(91.4,50.8)$ \\
7 & $93.1(60.0,96.0)$ & $87.4(92.8,72.1)$ & $86.2(92.4,66.7)$ \\
8 & $95.0(84.0,96.2)$ & $92.0(95.3,82.4)$ & $88.5(93.9,71.4)$ \\
9 & $95.4(80.0,97.0)$ & $90.8(94.8,79.4)$ & $89.7(93.9,76.2)$ \\
11 & $96.2(88.0,97.0)$ & $92.3(94.3,86.8)$ & $91.2(93.9,82.5)$ \\
12 & $96.6(88.0,97.5)$ & $95.4(96.4,92.7)$ & $93.9(96.0,87.3)$ \\
13 & $100(100,100)$ & $95.0(96.9,89.7)$ & 95.0 \\
15 & $100(100,100)$ & $98.9(99.5,97.1)$ & 94.0 \\
\hline
\end{tabular}

${ }^{1}$ Data presented as the percentage of correct diagnosis of the lameness model followed by sensitivity and specificity, respectively, in parentheses.

tion primarily improved model specificity and affected sensitivity at a much lower level. As a result, model accuracy and ROC increased principally through improvements in specificity (Table 2). Because of the low number of forelimb lameness in the data set, we were unable to conclusively show that the forelimb lameness model outperformed the cow level model.

\section{Effects of the LMV Transformation}

In Figures 1, 2, and $3(\mathrm{n}=261$ cows), veterinary clinical diagnosis ( 0 for sound and 1 for lame) was plotted against the model's predicted probability of lameness of a given cow for probabilities estimated with untransformed (Figure 1) and transformed LMV at 7 knots (Figure 2) and 15 knots (Figure 3). Of the 198 clinically lame cows (veterinary clinical diagnosis $=1$ ), use of untransformed LMV (Figure 1) resulted in the model predicting that 20 cows had $<50 \%$ probability of being lame, whereas 178 had $>50 \%$ probability of being lame. Of the 63 cows judged clinically sound (clinical diagnosis $=0$ ), 12 were determined by the model to have $<50 \%$ probability of being lame, and 51 were determined to have $>50 \%$ probability of being lame. Thus, of the 261 cows, 49 clinically sound cows were predicted by the model as lame, and 21 clinically lame animals were predicted by the model as sound.

Model performance using B-spline TLMV after 7 knots (Figure 2) showed greater agreement between the predicted probability of the model and the actual clinical diagnoses. Note the improved separation between animals judged by clinical evaluation as sound (0) and lame (1) cows. Of 198 clinically lame cows (clinical diagnosis $=1$ ) use of TLMV (Figure 2) resulted in the model predicting that 12 cows had $<50 \%$ probability of being lame, whereas 186 cows had $>50 \%$ probability of being lame. Of the 63 cows judged clinically sound (clinical diagnosis $=0$ ), 45 were determined by the model to have $<50 \%$ probability of being lame, and 18 were determined to have $>50 \%$ probability of being lame.

Model performance using B-spline TLMV after 15 knots (Figure 3) showed more accurate model performance. Of the 198 clinically lame cows (clinical diagnosis $=1$ ) use of spline-transformed LMV (Figure 3) after 15 knots resulted in the model predicting only 1 cow with $<50 \%$ probability of being lame and 197 cows with $>50 \%$ probability of being lame. Of 63 cows judged clinically sound (clinical diagnosis $=0$ ), all 63 were determined by the model to have $<50 \%$ probability of being lame.

\section{Optimal Choice of Knots}

The model evaluated hereafter was derived at the cow level using the TLMV as the independent explanatory variables and cow lameness diagnosis as the dependent variable. Model performance in Figure 4 was evaluated using the leave-one-out method. The percentage of correct lameness diagnosis was distributed across the number of knots for 1, 2, or 3 df (Figure 4). Correct diagnosis was assigned when the model and veterinary diagnoses agreed. Figure 4 demonstrated that model performance, using the TLMV, improved as the number of knots increased from 1 to 15 . The percentage correct diagnosis was $73.2 \%$ when the number of knots was 1 and exceeded $95.0 \%$ when the number of knots exceeded 13. Note the degrees of freedom appeared to have no effect on prediction accuracy. 


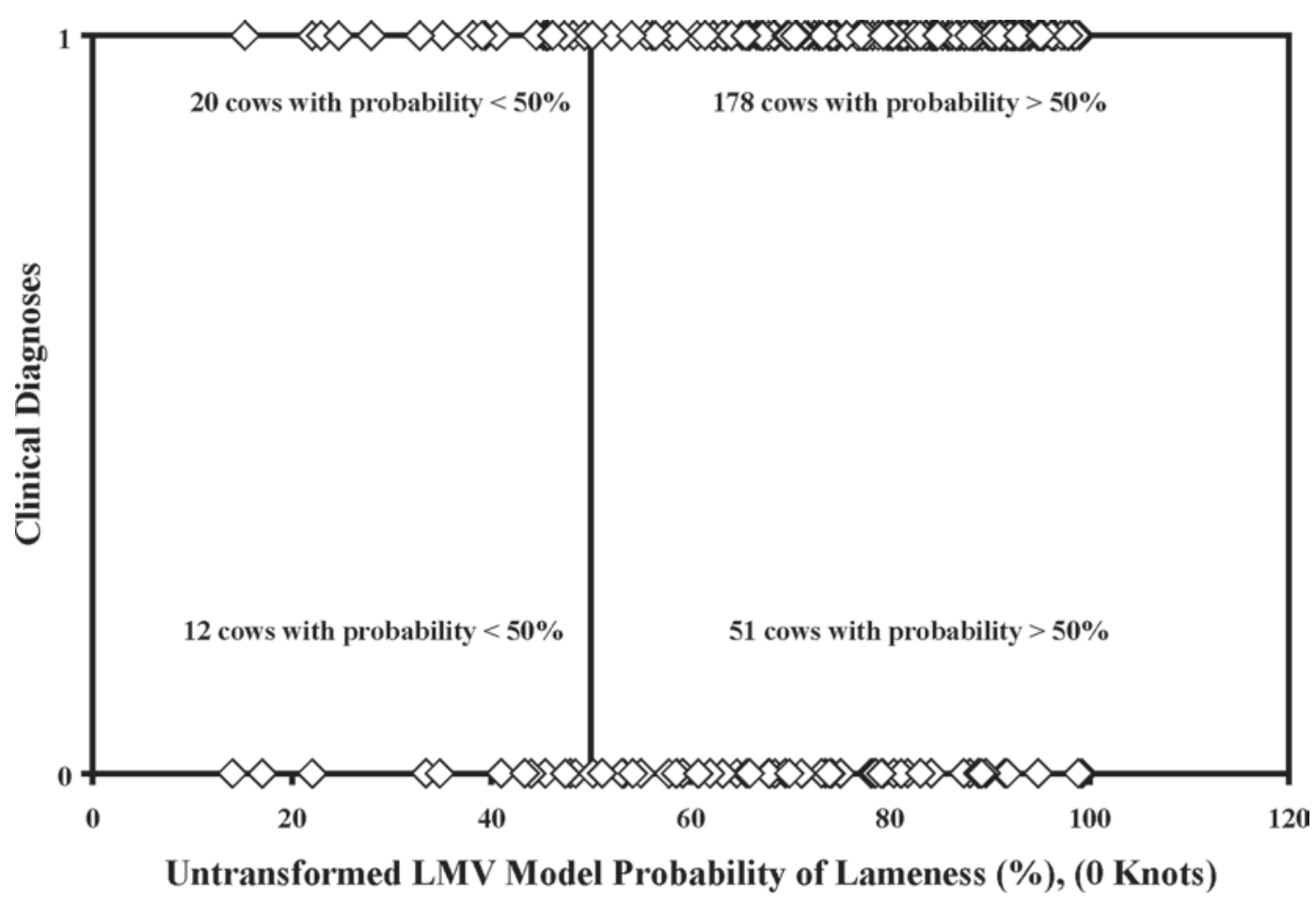

Figure 1. Clinical diagnoses versus the probability of being lame for the untransformed (knots $=0)$ limb movement variables (LMV) model. Clinical score of $0=$ clinically sound cow; $1=$ clinically lame cow. Model-predicted probability $\leq 50 \%=$ model prediction of sound; modelpredicted probability $\geq 50 \%=$ model prediction of lame. Each individual cow is represented by $\diamond$.

The ROC curve (Duh, 1998) was employed to evaluate lameness model performance. The ROC curves were es-

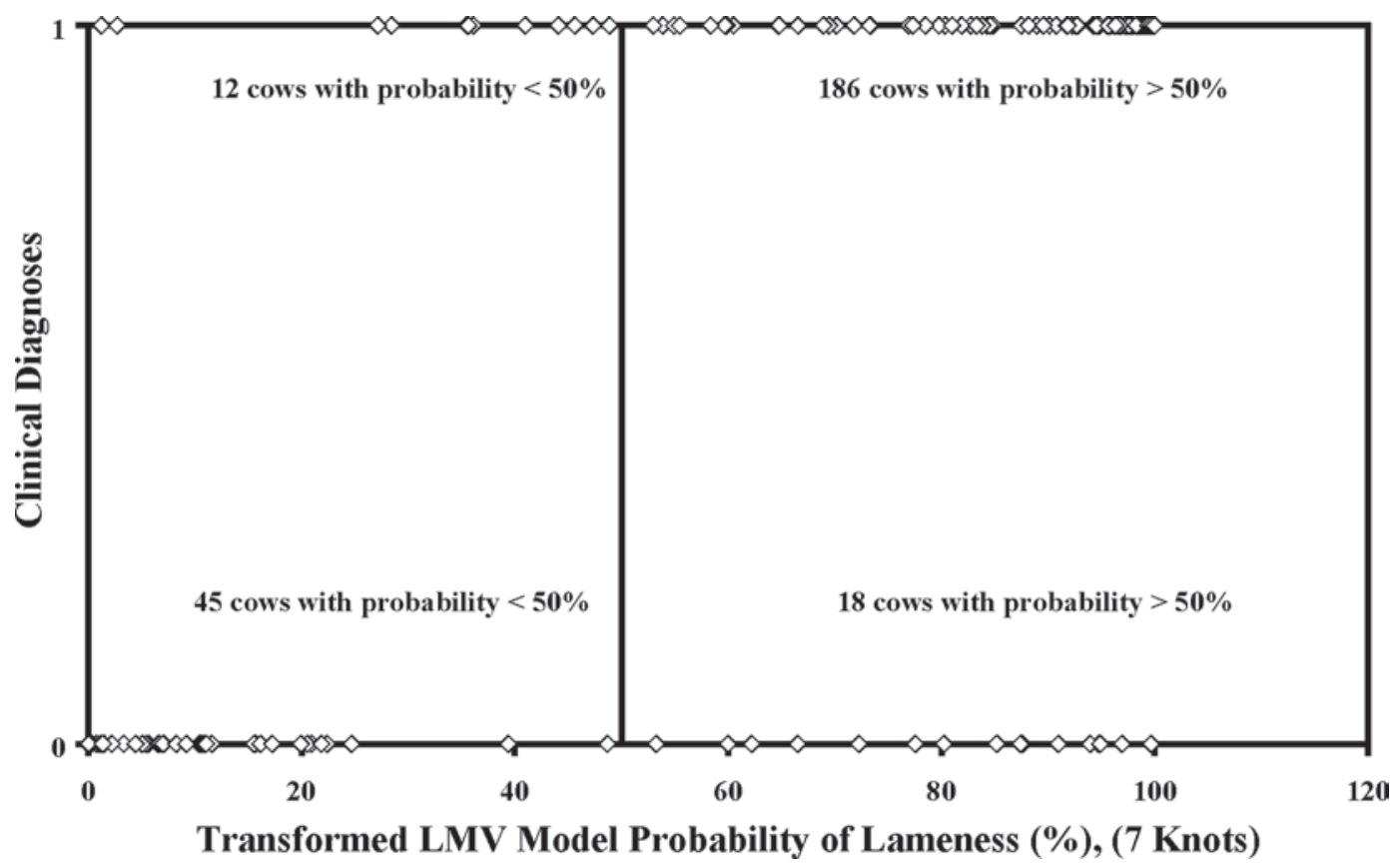

Figure 2. Clinical diagnoses versus the probability of being lame for the transformed (knots $=7$ ) limb movement variables (LMV) model. Clinical score of $0=$ clinically sound cow; $1=$ clinically lame cow. Model-predicted probability $\leq 50 \%=$ model prediction of sound; modelpredicted probability $\geq 50 \%=$ model prediction of lame. Each individual cow is represented by $\diamond$. 


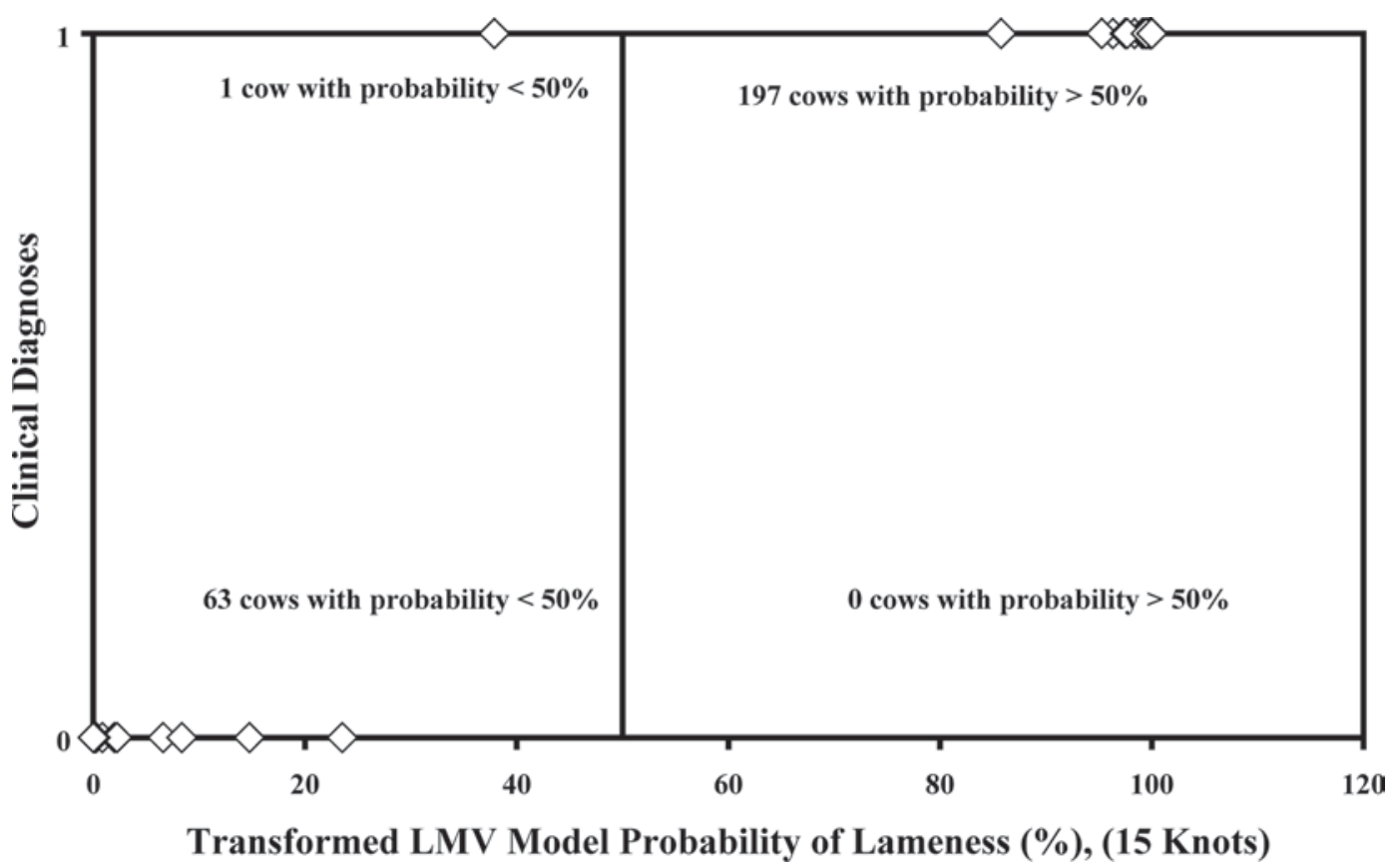

Figure 3. Clinical diagnoses versus the probability of being lame for the transformed (knots $=15)$ limb movement variables (LMV) model. Clinical score of $0=$ clinically sound cow; $1=$ clinically lame cow. Model-predicted probability $\leq 50 \%=$ model prediction of sound; modelpredicted probability $\geq 50 \%=$ model prediction of lame. Each individual cow is represented by $\diamond$.

tablished for models generated with spline-transformed LMV, when $\mathrm{df}=1$, and the number of knots varied from 0 to 15 (Figure 5). Figure 5 and Table 2 clearly demonstrated that the AUC increased with increased number of knots. At knots >10, model performance approached perfection with sensitivity, specificity, and AUC values all close to 1 .

\section{Testing the Stability of the Lameness Model at the Cow Level}

Modeling with spline-transformed data with large number of knots resulted in accuracy approaching $100 \%$. This suggested larger number of knots resulted in better model performance. To further address this issue, stability tests were performed on the lameness model by exposing the measured LMV to additional small perturbations (ranging from 0.5 to $5.0 \%$ ) that were superposed on the measurement noise. When perturbations were added to generate the simulated cow database, perturbations were constrained from changing the LMV characteristics of typical sound $(0 \%$ perturbation, Table 3) and lame (0\% perturbation, Table 4) cows. The original and simulated LMV values generated by perturbations of 0.5 to $5.0 \%$ of sound and lame animals are depicted in Tables 3 and 4. Perturbations greater than $1.0 \%$ generated LMV values with ranges that exceeded the ranges measured on the farm by more than
$5.0 \%$. Therefore, to retain the statistical characteristics of typical sound and lame cows, the perturbation level was limited in Equation 4 to 1\%. Figures 6(A) and (B) depict the sensitivity, specificity, and correct diagnosis when the LMV perturbations were 0.5 and $1.0 \%$, respectively. The model performances in Figures 6(A) and (B) were plotted as functions of number of knots. Note that up to a critical knots value, model specificity greatly improved, whereas model sensitivity modestly improved. Clearly, improvements in model specificity and sensitivity were realized across the 2 different data set perturbations. Furthermore, Figures 6(A) and (B) demonstrated that different levels of LMV perturbation resulted in different optimal number of knots. Deterioration in specificity (Figure $6 \mathrm{~A}$ ) at $0.5 \%$ LMV perturbation and sensitivity (Figure 6B) at 1.0\% perturbation with $\geq 7$ knots eroded model accuracy in ways not observed in the unperturbed data set (Table 2).

\section{DISCUSSION}

Early field trials indicated model accuracy was limited and marked by low ROC values (Bicalho et al., 2007a). We repeated the observations using the same system in different groups of animals and found diagnostic accuracy was marked by low specificity, high sensitivity, and moderately low ROC values. In these trials, use of untransformed LMV in limb or cow level models was 


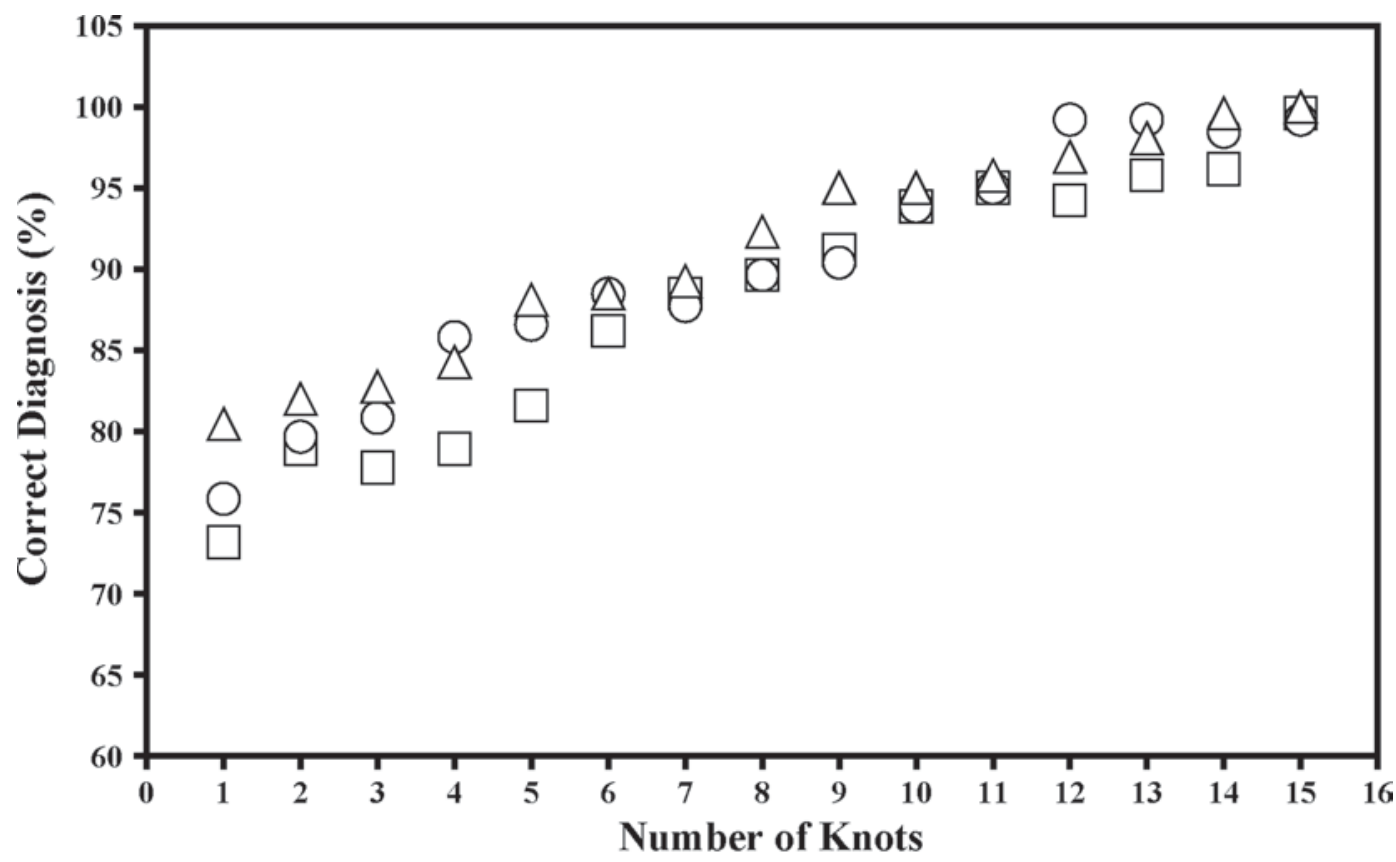
$\square$ Degree $=1$
O Degree $=2$
$\triangle$ Degree $=3$

Figure 4. Percentage of correct diagnoses of lameness versus number of knots for 3 levels of degrees of freedom (Degree).

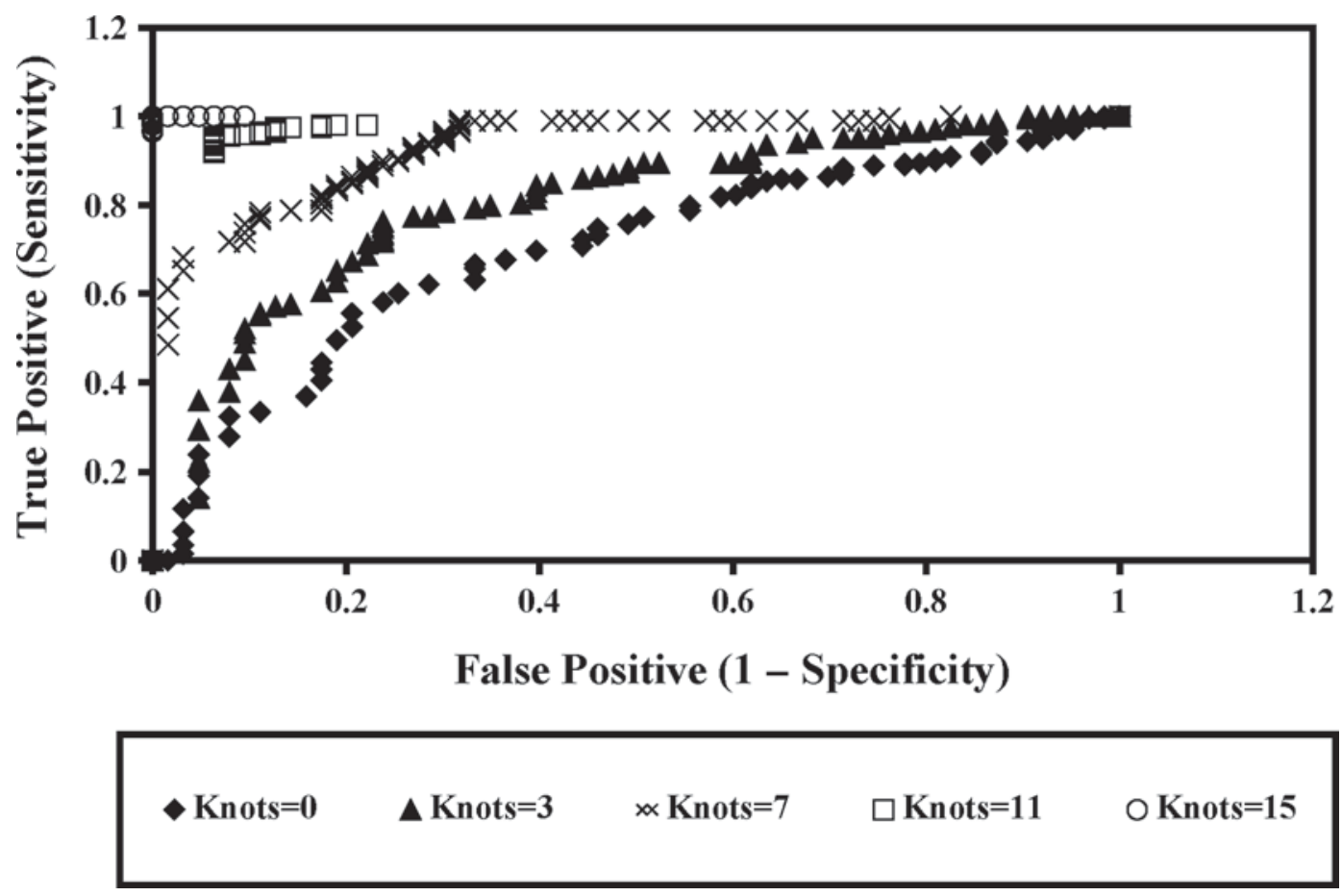

Figure 5. Receiver operating characteristic (ROC) curves for transformed limb movement variables (LMV) with number of knots varying from 0 (untransformed) to 15. The area under the curve increased with increased knot numbers. 
Table 3. Ranges of the measured ( $0 \%$ perturbation) limb movement variables (LMV) 1 to 16, and ranges of the simulated LMV with different perturbation levels (0.5 to $5 \%$ ) for sound cows ${ }^{1}$

\begin{tabular}{|c|c|c|c|c|c|c|c|}
\hline \multirow[b]{2}{*}{ Item } & \multicolumn{7}{|c|}{ Perturbation level, \% } \\
\hline & 0 & 0.5 & 1 & 2 & 3 & 4 & 5 \\
\hline LMV3 & 0.300 & 0.305 & 0.315 & 0.313 & 0.314 & 0.341 & 0.347 \\
\hline LMV4 & 0.410 & 0.412 & 0.417 & 0.442 & 0.436 & 0.448 & 0.445 \\
\hline LMV5 & 0.280 & 0.290 & 0.300 & 0.300 & 0.347 & 0.334 & 0.372 \\
\hline LMV8 & 0.400 & 0.401 & 0.405 & 0.413 & 0.477 & 0.459 & 0.548 \\
\hline LMV9 & 0.330 & 0.336 & 0.342 & 0.341 & 0.363 & 0.379 & 0.370 \\
\hline LMV10 & 0.250 & 0.251 & 0.257 & 0.253 & 0.280 & 0.261 & 0.280 \\
\hline LMV11 & 0.200 & 0.202 & 0.202 & 0.207 & 0.229 & 0.220 & 0.257 \\
\hline LMV12 & 0.390 & 0.393 & 0.407 & 0.415 & 0.421 & 0.461 & 0.463 \\
\hline LMV13 & 0.340 & 0.345 & 0.348 & 0.368 & 0.363 & 0.385 & 0.345 \\
\hline LMV14 & 0.280 & 0.284 & 0.285 & 0.317 & 0.305 & 0.298 & 0.354 \\
\hline
\end{tabular}

${ }^{1}$ Values in bold depict perturbed LMV ranges that exceed the corresponding measured LMV ranges by less than $5 \%$.

marked by an acceptable ability to correctly diagnose lameness when present, but an unacceptably low ability to diagnose soundness when present. Altogether, specificity and AUC values for the cow level model indicated use of untransformed LMV inputs were of marginal value as predictors of lameness. The explanations underlying these problems are not clear. Problems inherent in subjective scoring of clinical lameness or differences across farm environments could have contributed to these problems. The relationships between clinical lameness and LMV may be inconsistent across different populations of animals in different farm environments (Rajkondawar et al., 2002). The original logistic model might have adequately explained the relationship between LMV and clinical lameness score in a particular group of LMV generated from one group of animals, but the same model may have been unable to capture the relationship across a broader range of LMV from diverse groups of animals.

Although the lameness model captured in Equation [1] was moderately successful, the level of model accuracy suggested that the full information contained in the data may not have been captured. Thus, the challenge was to obtain a model that exploited the nonlinear structure of the problem, yet maintained the logistic predictions. An overview of the general classes of models available for our data structure is first provided. Note that Equation [1] can be rewritten as

Table 4. Ranges of the measured ( $0 \%$ perturbation) limb movement variables (LMV) 1 to 16, and ranges of the simulated LMV with different perturbation levels (0.5 to $5 \%$ ) for lame cows ${ }^{1}$

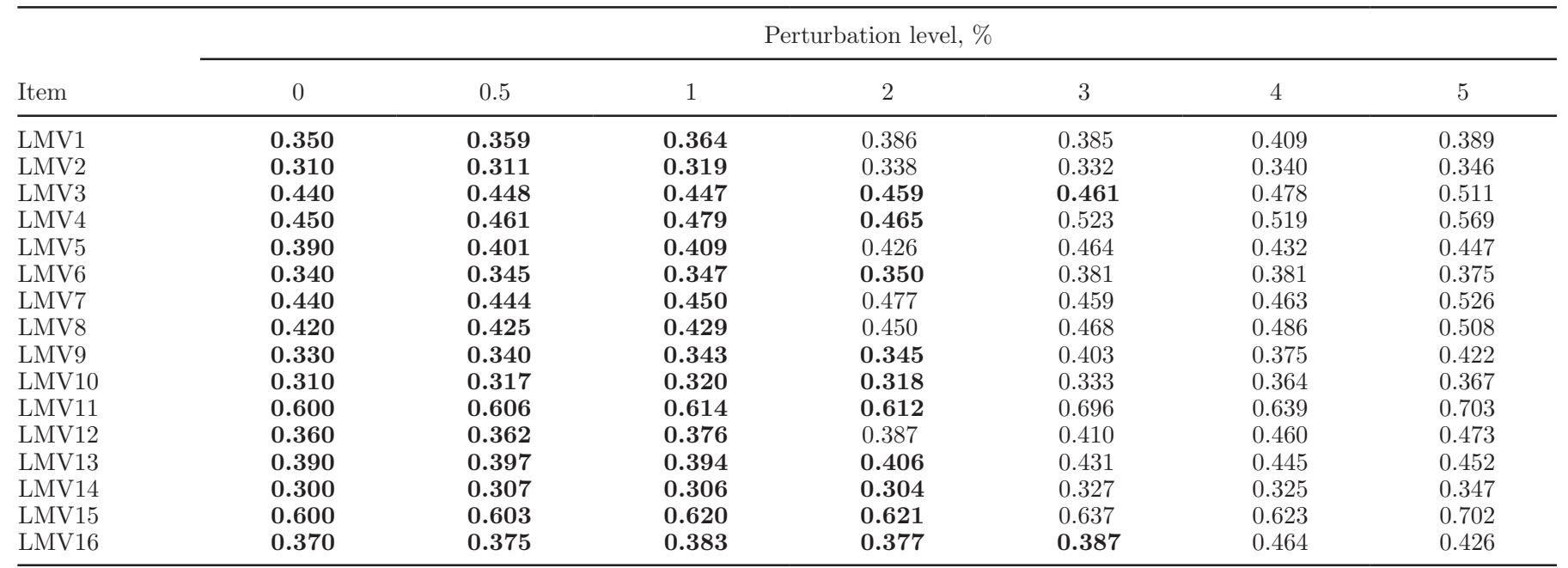

\footnotetext{
${ }^{1}$ Values in bold depict perturbed LMV ranges that exceed the corresponding measured LMV ranges by less than $5 \%$.
} 
A

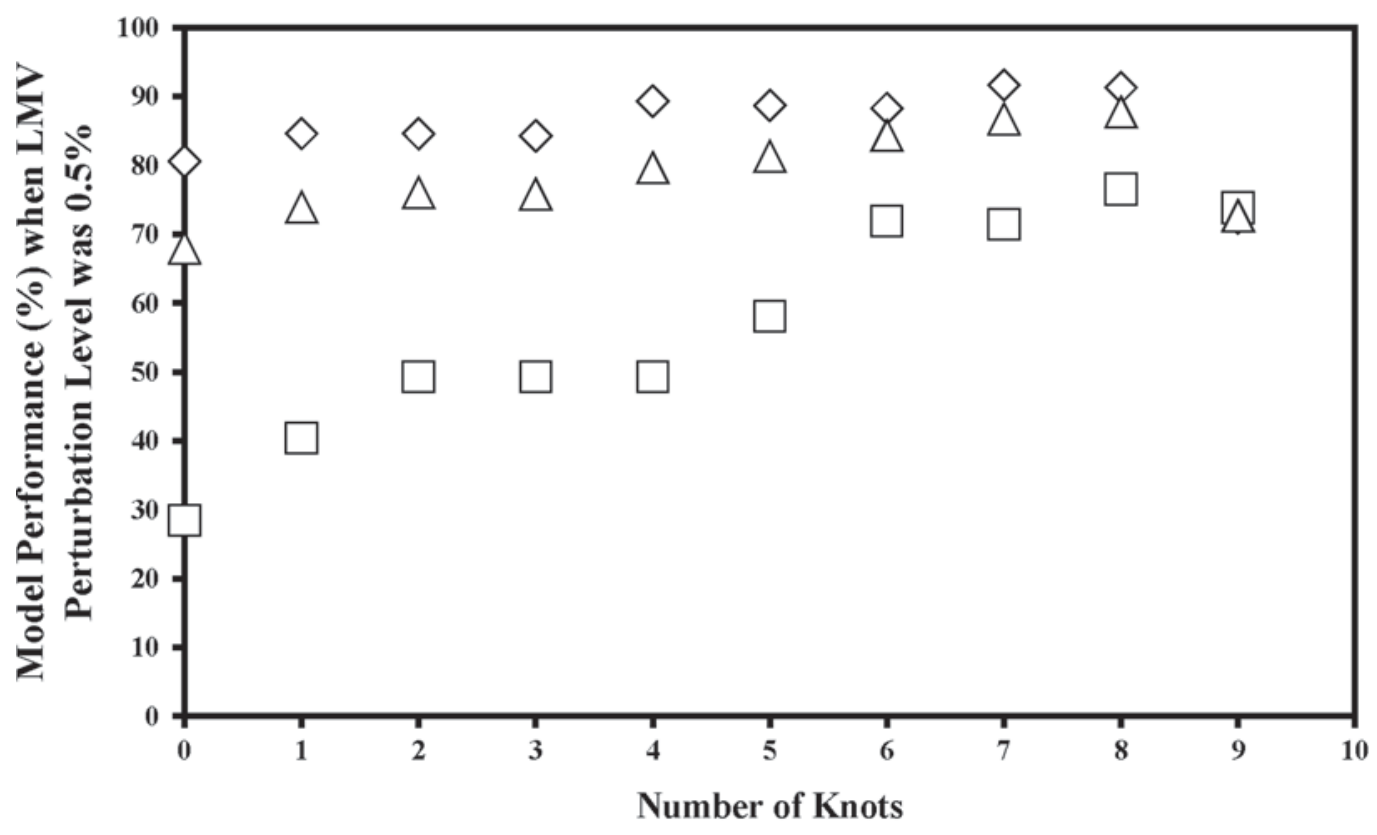

$\diamond$ Sensitivity $\square$ Specificity $\quad \Delta$ Correct Diagnosis

B

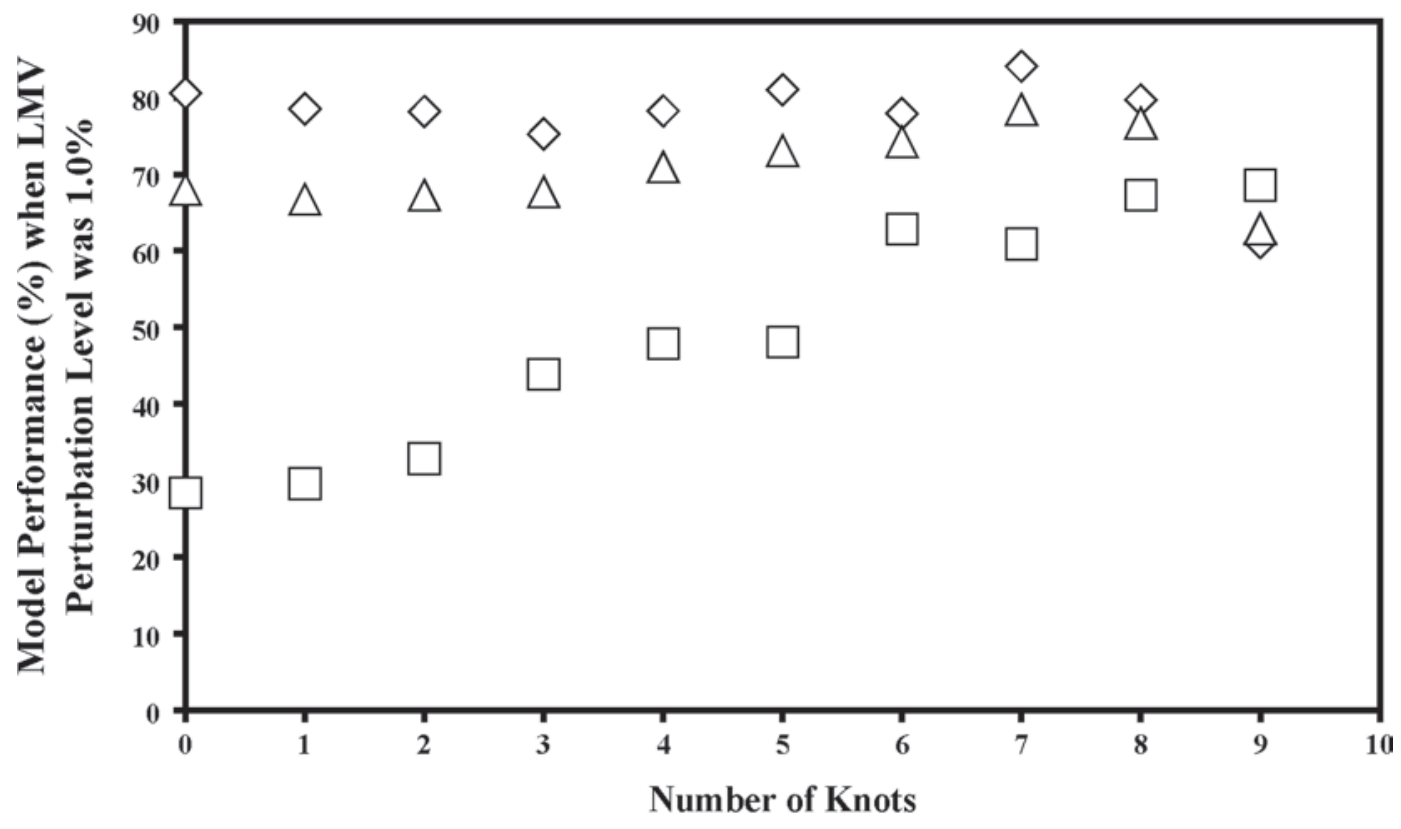

$\diamond$ Sensitivity

$\square$ Specificity

$\triangle$ Correct Diagnosis

Figure 6. Percentages of correct diagnoses, sensitivity, and specificity as functions of the number of knots for perturbed limb movement variables (LMV) levels. A) Perturbed LMV level of 0.5\%; B) perturbed LMV level of $1.0 \%$. 


$$
P(\text { Lameness }=1)=\eta(x),
$$

where $\eta(x)$ is the logistic function $\frac{e^{x}}{1+e^{x}}$, and $x=\beta_{0}+$ $\Sigma \beta_{i} L M V_{i}$. In general, $\eta(x)$ can be any function that maps a linear combination of the LMV into the probability of lameness. This class of models is called a generalized linear model (GLM) and the function $\eta$ is the link function. The logistic regression function used in Equation [1] is the most common link function. Still, there are many other choices of link functions (McCullagh and Nelder, 1989). The key aspect of this model was that the predictor variables were entered into the link function linearly. One way to expand the scope of the model is to enter powers of predictor variables so that they enter the link function via polynomials. Yet, in our case, this did not make a remarkable difference in the model performance. Another way of expanding the scope of the GLM was to estimate the link function. This led to a class of models in the form of Equation [5], where both $\eta(x)$ and the $\beta$-coefficients were estimated. Usually, $\eta(x)$ is estimated by nonparametric smoothing methodology. These models are known as single index models (Carroll, et al., 1997). The drawback of this method is that $\eta(x)$ is difficult to constrain to take values in the interval $(0,1)$ so that the predictions are legitimate probability values. A generalization of the usual linear models, known as generalized additive models (GAM) was introduced by Hastie and Tibshirani (1990). There, the predictor variables were transformed and estimated nonparametrically, one at a time, to build a linear model. Thus, GAM is of the form

$$
P(\text { Lameness }=1)=\beta_{0}+\Sigma \beta_{i} \eta_{i}(L M V) .
$$

Because the right-hand side is a linear combination of transformed predictor variables, restricting the predicted values to an acceptable range of 0 to 1 would not be possible. Thus, a combination of the GLM and GAM was considered, the logistic link function was utilized with a GAM approach to transform the predictor variables. The models considered here were of the form:

$$
P(\text { Lameness }=1)=\frac{e^{\beta_{0}+\Sigma \beta_{i} \eta_{i}(L M V)}}{1+e^{\beta_{0}+\Sigma \beta_{i} \eta_{i}(L M V)}} .
$$

Thus, both $\eta$-transformations and $\beta$-coefficients needed to be estimated. Our approach was to first obtain transformations of the LMV (possibly nonlinear) and subsequently utilized the TLMV in a logistic regression model.
Further efforts to develop automated mechanisms of lameness detection were pursued by testing the hypothesis that B-spline transformation would increase the ability of the logistic model to distinguish lame from sound cows. Indeed, spline-transformed LMV inputs improved model performance as the number of knots employed in the transformation was increased. The data clearly indicated transformation improved model performance principally by targeting specificity. There was a smaller improvement in sensitivity as the model more and more closely fit the data set. Thus, transformation improved the most inaccurate component in the original (0 knot) lameness detection model. Indeed, the fit became so accurate that the model approached the standards for the perfect diagnostic test (specificity $=$ sensitivity $=\mathrm{AUC}=1$ ). At knots $=15$, model accuracy, sensitivity, and specificity were 99.6, 99.5, and $100 \%$, respectively, which represented excellent performance by any measure, because increased model capability of discriminating between lame and sound cows resulted in very high accuracy. The results demonstrated spline functions and logistic regression could work in combination to develop improved predictive models of lameness. In accordance with these results, Tang et al. (2008) recently demonstrated spline transformation and logistic regression generated a model that successfully predicted the presence of amyotrophic lateral sclerosis in rodents.

Although spline transformation may have improved lameness detection, the approach to modeling lameness may need to incorporate additional avenues of independent (LMV) as well as dependent (clinical lameness score) input. Limb movement variable inputs employed in the current regression model were key measures of kinetic events in lame and sound limbs. Because Flower and Weary (2006) showed kinematic variables discriminated between sound and lame limbs, it is conceivable that kinematic inputs could have enhanced the discriminating abilities of models based solely upon kinetic variables. Perhaps an even greater problem eroding model accuracy stemmed from the unreliable nature of lameness scoring employed to generate the dependent variable. Even with very experienced, welltrained individuals, agreement across lameness scores among observers remained sufficiently low, which probably compromised most modeling efforts. Engel et al. (2003) and Flower and Weary (2006) showed training and experience improved scoring agreement, but the improvement may be insufficient for modeling purposes. Furthermore, (Engel et al., 2003) found across-observer agreement between consecutive scores was low and unlikely conducive to accurate modeling development. Recently, (Bicalho et al., 2007a) used measures of pain to validate visual scoring and showed gait scores $\geq 3$ could 
locate painful claw lesions with modest sensitivity and higher specificity. Moreover, objective measures of claw pain clearly showed a complicated relationship existed between claw pain and visual gait score (Dyer et al., 2007), and the relationship changed depending upon lesion type and time of trimming relative to assessment of claw pain and gait scores. For purposes of model development, the low sensitivity of visual scoring (Bicalho et al., 2007a), coupled with inconsistency among observers (Engel et al., 2003; Bicalho et al., 2007a), underscored the necessity to improve estimated values of the dependent variable. Clearly, more-objective measures of clinical lameness could be expected to greatly improve dependent variable estimates. Recognizing this problem, clinical scores employed in the current report incorporated objective measures of pain and objective measures of lesion while collapsing subjective measures of lameness score into 2 levels for the dependent variable. This approach may have diminished the error in the dependent variable and contributed to improved model accuracy.

In conclusion, the spline transformation of the LMV enhanced the accuracy of the lameness predictions and improved model sensitivity and specificity. Small perturbations in LMV inputs showed model stability depended upon the number of knots employed in the transformation across modified data sets. Regression techniques and spline transformation combined to improve model accuracy and generated avenues that might be useful for improved model performance across animal populations.

\section{ACKNOWLEDGMENTS}

The authors acknowledge the support of Tom England and Tom Frey from Frey Dairy Inc. (Lancaster, PA) and the Zimmerman family from Meadowview Farms (Berks County, PA). This research was partially supported by USDA-SBIR grants 2004-33610-14360 and 2005-03204.

\section{REFERENCES}

Amory, J. R., Z. E. Barker, J. L. Wright, S. A. Mason, R. W. Blowey and L. E. Green. 2008. Associations between sole ulcer, white line disease and digital dermatitis and the milk yield of 1824 dairy cows on 30 dairy cow farms in England and Wales from February 2003-November 2004. Prev. Vet. Med. 83:381-391.

Animal Welfare Foundation. 1993-1994. Annual Review. British Veterinary Assoc., London, UK.

Bicalho, R. C., S. H. Cheong, G. Cramer, and C. L. Guard. 2007a. Association between a visual and an automated locomotion score in lactating Holstein cows. J. Dairy Sci. 90:3294-3300.

Bicalho, R. C., F. Vokey, H. N. Erb, and C. L. Guard. 2007b. Visual locomotion scoring in the first seventy days in milk: Impact on pregnancy and survival. J. Dairy Sci. 90:4586-4591.

Bicalho, R. C., L. D. Warnick, and C. L. Guard. 2008. Strategies to analyze milk losses caused by diseases with potential incidence throughout the lactation: A lameness example. J. Dairy Sci. 91:2653-2661.
Booth, C. J., L. D. Warnick, Y. T. Grohn, D. O. Maizon, C. L. Guard, and D. Janssen. 2004. Effect of lameness on culling in dairy cows. J. Dairy Sci. 87:4115-4122.

Carroll, R. J., J. Fan, I. Gijbels, and M. P. Wand. 1997. Generalized partially linear single-index models. J. Am. Stat. Assoc. 92:477489.

Duh, M. S., A. M. Walker, M. Pagano, and K. Kronlund. 1998. Prediction and cross validation of neural networks versus logistic regression: Using hepatic disorders as an example. Am. J. Epidemiol. 147:407-413.

Dyer, R. M., N. K. Neerchal, U. Tasch, Y. Wu, P. Dyer, and P. G. Rajkondawar. 2007. Objective determination of claw pain and its relationship to limb locomotion score in dairy cattle. J. Dairy Sci. 90:4592-4602.

Engel, B., G. Bruin, G. Andre, and W. Buist. 2003. Assessment of observer performance in a subjective scoring system: Visual classification of the gait of cows. J. Agric. Sci. 140:317-333.

Espejo, L. A., M. I. Endres, and J. A. Salfer. 2006. Prevalence of lameness in high-producing Holstein cows housed in freestall barns in Minnesota. J. Dairy Sci. 89:3052-3058.

Eubank, R. L. 1999. Nonparametric Regression and Spline Smoothing. 2nd ed. Marcel Dekker Inc., New York.

Flower, F. C., and D. M. Weary. 2006. Effect of hoof pathologies on subjective assessments of dairy cow gait. J. Dairy Sci. 89:139146.

Garbarino, E. J., J. A. Hernandez, J. K. Shearer, C. A. Risco, and W. W. Thatcher. 2004. Effect of lameness on ovarian activity in postpartum Holstein cows. J. Dairy Sci. 87:4123-4131.

Green, L. E., V. J. Hedges, Y. H. Schukken, R. W. Blowey, and A. J. Packington. 2002. The impact of clinical lameness on the milk yield of dairy cows. J. Dairy Sci. 85:2250-2256.

Hastie, T. J., and R. J. Tibshirani. 1990. Generalized Additive Models. Chapman \& Hall/CRC, New York, NY.

Hosmer, D. W., and S. Lemeshow. 2000. Applied Logistic Regression. John Wiley and Sons Inc., New York, NY.

Kohavi, R. 1995. A study of cross-validation and bootstrap for accuracy estimation and model selection. Int. Joint Conf. Artificial Intelligence. Montréal, Québec, Canada.

McCullagh, P., and J. A. Nelder. 1989. Generalized Linear Models. 2nd ed. Chapman \& Hall, London, UK.

NRC. 2001. Nutrient Requirements of Dairy Cattle. 7th ed. Natl. Acad. Sci., Washington, DC.

SAS Institute. 2004. SAS/STAT 9.1 User's Guide. SAS Institute Inc. Cary, NC.

Rajkondawar, P. G., A. M. Lefcourt, N. K. Neerchal, R. M. Dyer, M. A. Varner, B. Erez, and U. Tasch. 2002. The development of an objective lameness scoring system for dairy herds: Pilot study. Trans. ASAE 45:1123-1125.

Rajkondawar, P. G., U. Tasch, A. M. Lefcourt, B. Erez, R. M. Dyer, and M. A. Varner. 2001. A system for identifying lameness in dairy cattle. Appl. Eng. Agric. 18:87-96.

Schumaker, L. L. 2007. Spline Functions: Basic Theory. 3rd ed Cambridge University Press, Cambridge, UK.

Shearer, J., D. Anderson, W. Ayars, E. Belknap, S. Berry, C. Guard, K. Hoblet, E. Hovingh, G. Kirksey, A. Langill, A. Mills, D. Miskimins, J. Wasson, N. Cook, E. Garret, D. G. Hostetler, and L. Schugel. 2004. A record-keeping system for capture of lameness and foot-care information in cattle. Bovine Pract. 38:83-92.

Tang, W., U. Tasch, N. K. Neerchal, L. Zhu, and P. Yarowsky. 2008. Measuring early pre-symptomatic changes in locomotion of SOD1G93A rats - A rodent model of amyotrophic lateral sclerosis. J. Neurosci. Methods 176:254-262

Raven, E. T. 1985. The principals of claw trimming. Vet. Clin. North Am. Food Anim. Pract. 1:93-107.

USDA. 2008. Dairy 2007. Part II. Changes in the U.S. Dairy Cattle Industry, 1991-2007. USDA-NAHMS, Fort Collins, CO.

Whay, H. R., D. C. Main, L. E. Green, and A. J. Webster. 2003 Assessment of the welfare of dairy cattle using animal-based measurements: Direct observations and investigation of farm records. Vet. Rec. 153:197-202. 